\title{
Laparoscopic Pyelolithotomy: Initial Experience with 62 Patients
}

\section{Cyril Kamadjou',2, Annie Kameni', Dolly Bilonda Kolela1, Bertin Njinou Ngninkeu, Fru Angwafor ${ }^{3}$}

\author{
${ }^{1}$ Medical and Surgical Center of Urology, Douala, Cameroon \\ ${ }^{2}$ Department of Surgery and Specialities, Faculty of Medicine and Pharmaceutical Sciences, University of Douala, \\ Douala, Cameroon \\ ${ }^{3}$ Coordinator Urology Residency Program, Faculty of Medicine and Biomedical Sciences, University of Yaounde, \\ Yaounde, Cameroon \\ Email: cyrkamadjou@yahoo.fr
}

How to cite this paper: Kamadjou, C., Kameni, A., Kolela, D.B., Ngninkeu, B.N. and Angwafor, F. (2022) Laparoscopic Pyelolithotomy: Initial Experience with $62 \mathrm{~Pa}$ tients. Open Journal of Urology, 12, 74-82. https://doi.org/10.4236/oju.2022.121008

Received: December 17, 2021

Accepted: January 21, 2022

Published: January 24, 2022

Copyright (C) 2022 by author(s) and Scientific Research Publishing Inc. This work is licensed under the Creative Commons Attribution International License (CC BY 4.0).

http://creativecommons.org/licenses/by/4.0/

(c) (†) Open Access

\begin{abstract}
Objective: To evaluate the role of the laparoscopic approach in the management of pelvic calculi in a urology centre in Douala, Cameroon. Materials and Methods: This was a retrospective study carried out from 2014 to 2019 involving 62 patients with calculi at the renal pelvis managed through laparoscopic pyelolithotomy. Results: We recruited 62 patients $(38$ men and 24 women) with a median age of 36 [25 - 48] years. Nephritic colic was the most frequently encountered presenting complaint (92\% of cases). In $56.45 \%$ of cases, the stone was on the left side of the body. Twelve patients had kidney acute failure at the time of diagnosis. Five patients received antibiotics for urinary tract infections before the operation. Before surgery, eight double J stents were placed in 8 patients; in five because of urinary tract infection and in three because of nephritic colic. In all the cases, the definitive diagnosis was made using abdominal CT scans. The median size of the calculi was 22 [17 - 28] $\mathrm{mm}$. Dilatation of the pyelocaliceal cavity was noticed in all the patients. The median surgery duration was 100 [90 - 120] minutes. Drainage of the urinary tract using a double $J$ stent was performed in all the patients, whereas percutaneous drainage was performed only in $9.67 \%$ of cases. There was no case of conversion to open surgery. Control ultrasound performed one month after the removal of the double J stent did not reveal any residual stones in the pyelocaliceal cavities. Conclusion: Laparoscopic pyelolithotomy is a mini-invasive technique that is adequate for the management of voluminous pelvic calculi.
\end{abstract}

\section{Keywords}

Laparoscopic Pyelolithotomy, Pelvic Calculi, Cameroon 


\section{Introduction}

Different surgical techniques have been established for the management of different types of kidney stones, and the choice of a technique depends on stone location and distribution. These techniques include non-invasive and minimally invasive modalities such as ureteroscopy (URS), extracorporeal shockwave lithotripsy (ESWL), percutaneous nephrolithotomy (PCNL), laparoscopy, and robotic surgery [1]. Where non-invasive or minimally invasive methods are either unavailable or fail, invasive procedures (including open surgery) can be employed. The American Urological Association (AUA) recommends watchful waiting for patients with uncomplicated ureteral stones with sizes $\leq 10 \mathrm{~mm}$, URS for patients with mid- or distal ureteral stones who require intervention and for patients with suspected cystine or uric acid ureteral stones in whom medical expulsive therapy (MET) as a treatment modality for adult patients with ureteral stones fails. In the case of adult patients with renal stones, the AUA recommends ESWL or URS for symptomatic patients with a total non-lower pole renal stone burden $<20 \mathrm{~mm}$ and PCNL for symptomatic patients with a total renal stone burden $>20 \mathrm{~mm}$ [1]. Laparoscopic pyelolithotomy (LPL) is an important alternative to percutaneous nephrolithotomy (PCNL) in the management of large $(\geq 20 \mathrm{~mm}$ ) renal calculi; however, the latter technique remains the gold standard [2]. LPL is usually considered in patients who have renal anomalies, are poorly compliant, and have a large single renal-pelvic calculus [3]. LPL could follow a transperitoneal or retroperitoneal approach, and is usually performed under general anaesthesia. The transperitoneal approach involves insufflating the abdomen with carbon dioxide and making several small abdominal incisions. In the retroperitoneal approach, a small incision is made in the back and a dissecting balloon is inserted to create a retroperitoneal space. The stone is accessed through an incision in the renal pelvis (pyelotomy). Once the stone is removed, the pyelotomy incision is usually closed using sutures, with or without a stent [4]. LPL, like any other surgical procedure, is prone to complications. Potential complications of this technique are either associated with the removal of the stone or with the closure of the pyelotomy incision [5]. When laparoscopic pyeloplasty with concomitant pyelolithotomy was performed on 15 patients, all of them experienced wall oedema and friability due to fluid irrigation. Also, in a series involving 16 patients who underwent laparoscopic transperitoneal pyelolithotomy as the first-line treatment for pelvic stones published by Meria et al., 14 of the 16 patients had urinary leakage as a complication [6] [7]. This technique is more commonly used in developed countries, and the procedure has even been described in great detail [8]. However, despite all the advantages of these laparoscopic techniques, they are far from being common practice in resource-limited settings like ours, which explains the paucity of studies on this topic in such settings. Thus, we carried out this study that aimed to evaluate the role of the laparoscopic approach in the management of pelvic calculi at the Centre medico-chirugicaled urologie in Douala, Cameroon. 


\section{Methods and Materials}

\subsection{Study Design and Study Participants}

This was a retrospective study over a period of six years (from January 2014 to December 2019) at the Centre medico-chirugicaled urologie, which is located in Bali, Douala. We consulted the clinical records of 62 patients with calculi at the renal pelvis who were treated through laparoscopic pyelolithotomy. We excluded patients with incomplete clinical records. Using pre-tested questionnaires, we collected data on patients' ages, genders, clinical profiles, relevant medical history, sizes of the calculi, localisation of the calculi as confirmed by imaging, and outcome of lithotripsy. All patients underwent abdominopelvic CT before the procedure to localise the stones, and they all had at least one ureteric stone (Figure 1 and Figure 2).

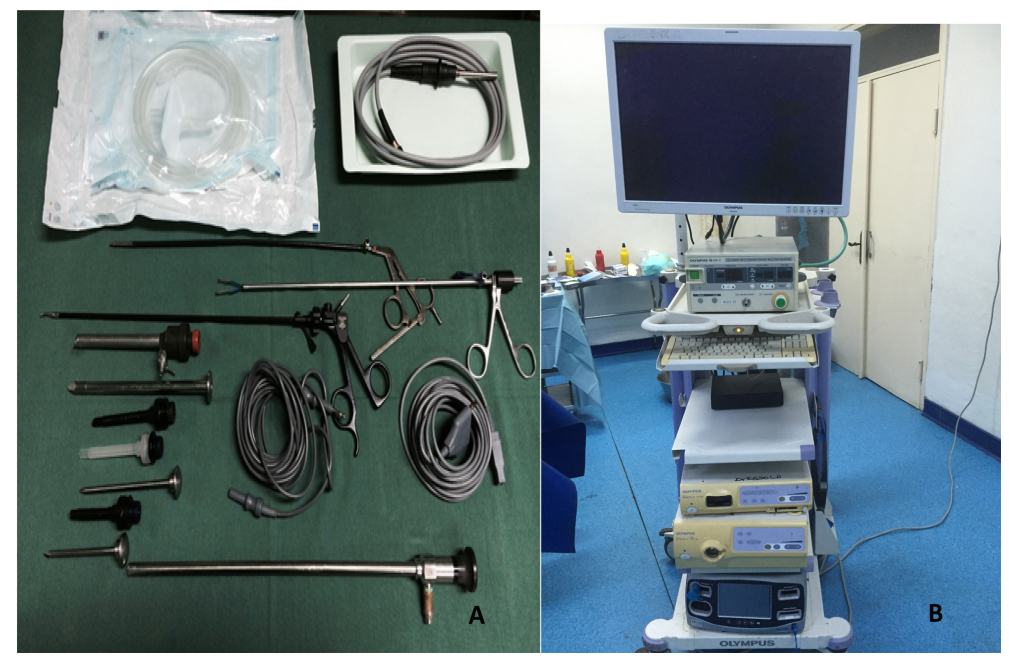

Figure 1. Laparoscopic devices (A) and laparoscopic tower (B).

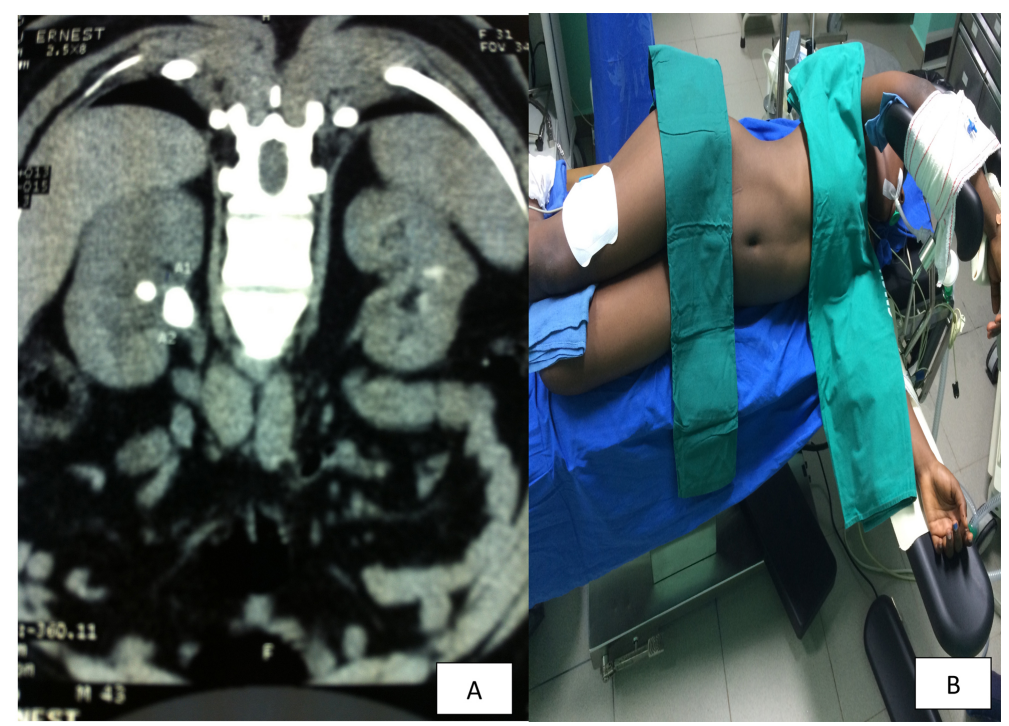

Figure 2. CT scan showing obstructive right renal pelvis stone (A) and patient placed in the left lateral position prior to surgical intervention (B). 


\subsection{Pre-Operative and Intraoperative Procedures}

All patients also consulted an anaesthesiologist anddida pre-operative workup, which included a full blood count, urea and creatinine, clotting profile, and urine analysis with culture and antibiotic susceptibility profiling. Those with confirmed urinary tract infections prior to the intervention were treated as per the results of culture and antibiotic susceptibility profiles. A second-generation cephalosporin was administered to all patients without confirmed urinary tract infections as a prophylactic antibiotic. All surgical procedures were performed under general anaesthesia. The patients were placed in the lateral position and the renal pelvis accessed transperitoneally. After inserting the abdominal trocars, pneumoperitoneum was achieved, and the kidney or ureter was exposed. The renal pelvis and ureter were identified, dissected, and opened. The stones were extracted using rigid laparoscopic forceps. The renal pelvis and ureter were sutured following the placement of an antegrade ureteral double J stent. The stones were then removed from the peritoneal cavity. In six patients, the procedure ended with the placement of an intraperitoneal drain. All the 62 participants were operated upon by the same two surgeons. Using the visual analogue scale (VAS), we classified the pain felt by the patients into either mild (VAS score < 4 ), moderate (VAS score ranging from 4 to 8 ), or severe (VAS score $>8$ ).

\subsection{Data Management}

The data collected from patients' clinical records were entered into Microsoft Excel 2016 and then exported to Epi info 7 for analysis. Continuous data are presented as mean values and standard deviations (for normally distributed data) and medians with interquartile ranges (for skewed data). On the other hand, categorical data are presented as frequencies and percentages.This study was approved by the institutional review board of the Faculty of Medicine and Pharmaceutical Sciences (FMPS) of the University of Douala and by the ethical committee of the Centre medico-chirugicale d urologie in Douala, Cameroon. The requirement for patients' informed consent was waived due to the retrospective nature of the study.

\section{Results}

Of the 62 patients we recruited in our study, 24 (38.71\%) were females and 38 (61.29\%) were males. The ages of the patients ranged from 7 years to 66 years, with a median age of 36 [25 - 48] years. As concerns the initial clinical presentations of the patients, $52(83.87 \%)$ patients presented with acute nephritic colic (ANC), 5 (8.06\%) presented with acute nephritic colic and sepsis, 3 (4.84\%) presented with abdominal pains, and $2(3.23 \%)$ presented with haematuria. The stones were located on the left side of the body in 35 (56.45\%) cases and on the right in 27 (43.55\%) cases. Fifty-one (82.26\%) patients experienced mild pain, 10 (16.13\%) experienced moderate pain, while only one participant (1.61\%) experienced severe pain. Data on the clinical presentations of the study partici- 
pants can be seen in Table 1 .

All the patients were hospitalised and underwent LPL, which was successful in all 62 patients (100\% stone-free rate). Of the 62 patients, double J stents were placed preoperatively in $8(12.9 \%)$ patients. Percutaneous drainage was also performed in $8(12.9 \%)$ patients. Double J drainage was carried out in all 62 patients post-operatively. Complications occurred in 3 (4.84\%) of our patients, who were all males; two (66.67\%) of these complications were cases of colic, while one (33.33\%) was a case of a fistula.

The duration of the mini-invasive procedure ranged from 60 minutes to200 minutes with a median duration of 100 [90 - 120] minutes). The duration of hospitalization of the patients ranged from 1 day to 5 days, with a mean duration of $2.21 \pm 1.07$ days. The estimated blood loss during the intervention ranged from $50 \mathrm{ml}$ to $400 \mathrm{ml}$ with a median estimated blood loss of 110 [80 - 150] $\mathrm{ml}$. The duration for which the double J stent was left in place ranged from 6 days to 90 days, with a median duration of 18 [15 - 21] days. The drains were left in place for durations ranging from 0 days to 3 days. The drains were removed on day 0 for 55 (88.71\%) participants, on day 1 for 1 (1.61\%) participant, on day 2 for $5(8.06 \%)$ participants, and on day 3 for 1 (1.61\%) participant. The details of the surgical procedure and postoperative hospitalization of the study participants are presented in Table 2.

As for the paraclinical tests carried out, the imaging technique used to locate the stones in all 62 patients was the (anteroposterior) CT scan. All the stones were located in the renal pelvis. The sizes of the stones ranged from $14 \mathrm{~mm}$ to 37 $\mathrm{mm}$, with a median stone size of $22[17-28] \mathrm{mm}$. Urinalysis was performed in 5 (8.06\%) of the participants. Regarding culprit pathogens, no pathogen was identified in 57 (91.94\%) cases. The culprit pathogens were Escherichia coli, Klebsiella pneumoniae, and Proteus mirabilis in 1 (1.61\%) case each, and it was

Table 1. Clinical presentations of the study participants.

\begin{tabular}{lccc}
\hline \multicolumn{1}{c}{ VARIABLES } & MALES (\%) & FEMALES (\%) & TOTAL (\%) \\
\hline Clinical presentation & & & \\
$\quad$ Colic & $34(89.47)$ & $18(75)$ & $52(83.87)$ \\
Colic and sepsis & $4(10.53)$ & $1(4.17)$ & $5(8.06)$ \\
Abdominal pain & $0(0)$ & $3(12.5)$ & $3(4.84)$ \\
$\quad$ Haematuria & $0(0)$ & $2(8.33)$ & $2(3.23)$ \\
Laterality & & & \\
Left & $22(57.89)$ & $13(54.17)$ & $35(56.45)$ \\
$\quad$ Right & $16(42.11)$ & $11(45.83)$ & $27(43.55)$ \\
Degree of pain (VAS) & & & \\
$\quad$ Mild & $29(76.32)$ & $22(91.67)$ & $51(82.26)$ \\
$\quad$ Moderate & $8(21.05)$ & $2(8.33)$ & $10(16.13)$ \\
Severe & $1(2.63)$ & $0(0.0)$ & $1(1.61)$ \\
\hline
\end{tabular}


Table 2. Details of the surgical procedure and hospitalisation of the participants.

\begin{tabular}{|c|c|c|c|}
\hline VARIABLES & $\begin{array}{l}\text { MALES } \\
(\%)\end{array}$ & FEMALES (\%) & TOTAL (\%) \\
\hline \multicolumn{4}{|l|}{ Pre-op double J } \\
\hline Yes & $6(15.79)$ & $2(8.33)$ & $8(12.90)$ \\
\hline No & $32(84.21)$ & $22(91.67)$ & $54(87.10)$ \\
\hline \multicolumn{4}{|l|}{ Percutaneous drainage } \\
\hline Yes & $7(18.42)$ & $1(4.17)$ & $8(12.90)$ \\
\hline No & $31(81.58)$ & $23(95.83)$ & $54(87.10)$ \\
\hline \multicolumn{4}{|l|}{ Double J drainage } \\
\hline Yes & $38(100)$ & $24(100)$ & $62(100)$ \\
\hline No & $0(0)$ & $0(0)$ & $0(0)$ \\
\hline \multicolumn{4}{|l|}{ Complications } \\
\hline None & $35(92.11)$ & $24(100)$ & $59(95.16)$ \\
\hline Colic & $2(3.23)$ & $0(0)$ & $2(3.23)$ \\
\hline Fistula & $1(1.61)$ & $0(0)$ & $1(1.61)$ \\
\hline $\begin{array}{l}\text { Median duration of surgery } \\
\text { (minutes) }\end{array}$ & $\begin{array}{c}100 \\
{[90-120]}\end{array}$ & $\begin{array}{c}95 \\
{[80-120]}\end{array}$ & $\begin{array}{c}100 \\
{[90-120]}\end{array}$ \\
\hline $\begin{array}{l}\text { Mean duration of } \\
\text { hospitalization (days) }\end{array}$ & $2.29 \pm 1.14$ & $2.08 \pm 0.97$ & $2.21 \pm 1.07$ \\
\hline Median estimated blood & 110 & 110 & 110 \\
\hline loss $(\mathrm{ml})$ & {$[80-150]$} & {$[82.5-145]$} & {$[80-150]$} \\
\hline $\begin{array}{l}\text { Median duration of double } \mathrm{J} \\
\text { stent in the body (days) }\end{array}$ & $18[15-21]$ & $18[16.5-21]$ & $18[15-21]$ \\
\hline \multicolumn{4}{|l|}{$\begin{array}{l}\text { Duration of the drain in the } \\
\text { body }\end{array}$} \\
\hline Day 0 & $32(84.21)$ & $23(95.83)$ & $55(88.71)$ \\
\hline Day 1 & $1(2.63)$ & $0(0)$ & $1(1.61)$ \\
\hline Day 2 & $4(10.53)$ & $2(4.17)$ & $5(8.06)$ \\
\hline Day 3 & $1(2.63)$ & $0(0)$ & $1(1.61)$ \\
\hline
\end{tabular}

Streptococcus pyogenes in $2(3.23 \%)$ cases.

The serum creatinine levels of the participants ranged from $7 \mathrm{mg} / \mathrm{L}$ to 37 $\mathrm{mg} / \mathrm{L}$, with a median value of $10[9-12] \mathrm{mg} / \mathrm{L}$. The results of the paraclinical workup are presented in Table 3. 
Table 3. Paraclinical parameters of the study participants.

\begin{tabular}{lccc}
\hline \multicolumn{1}{c}{ VARIABLES } & MALES (\%) & FEMALES (\%) & TOTAL (\%) \\
\hline $\begin{array}{l}\text { Stone size }(\mathrm{mm}) \\
<20\end{array}$ & $16(42.11)$ & $9(37.5)$ & $25(40.32)$ \\
$20-29$ & $17(44.73)$ & $7(29.17)$ & $24(38.71)$ \\
$\geq 30$ & $5(13.16)$ & $8(33.33)$ & $13(20.97)$ \\
Urinalysis & & & $5(8.06)$ \\
$\quad$ Yes & $4(10.53)$ & $1(4.17)$ & $57(91.94)$ \\
$\quad$ No & $34(89.47)$ & $23(95.83)$ & $57(91.94)$ \\
Culprit pathogen & & & $1(1.61)$ \\
$\quad$ None & $34(89.47)$ & $23(95.83)$ & $1(1.61)$ \\
Escherichia coli & $1(2.63)$ & $0(0.0)$ & $1(1.61)$ \\
$\quad$ Klebsiella pneumoniae & $1(2.63)$ & $0(0.0)$ & $2(3.23)$ \\
$\begin{array}{l}\text { Proteus mirabilis } \\
\text { Streptococcus pyogenes }\end{array}$ & $1(2.63)$ & $0(0.0)$ & $10[9-12]$ \\
$\begin{array}{l}\text { Median serum } \\
\text { creatinine (mg/L) }\end{array}$ & $1(2.63)$ & $1(4.17)$ & \\
\hline
\end{tabular}

\section{Discussion}

Renal and ureteric stones are generally managed by URS, ESWL, PCNL, robotic surgery, and laparoscopic techniques. Laparoscopic techniques such as laparoscopic pyelolithotomy and laparoscopic ureterotomy are usually considered in patients who have renal anomalies, are poorly compliant, and have a large single renal-pelvic calculus [3]. The current study shows our experience in the management of 62 patients with renal calculi using laparoscopic pyelolithotomy.

A meta-analysis assessing the efficacy and safety of LPL versus percutaneous nephrolithotomy (PCNL) for the treatment of renal pelvic calculi $>20 \mathrm{~mm}$ revealed that LPL is associated with a significantly higher stone-free rate than PCNL [9]. Al-Hunayan et al. compared the outcome of LPL using the transperitoneal approach to that using the retroperitoneal approach and found out that compared to transperitoneal LPL, retroperitoneal LPL for large renal pelvic stones resulted in a shorter operative time, a shorter resumption time for normal oral intake, and a shorter hospital stay. There was no significant difference in the stone-free rate between the two approaches [10]. In our study, patients were treated using the transperitoneal approach as we were more familiar with this approach and have had few complications using it in the past.

In the present study, we obtained a stone-free rate of $100 \%$ irrespective of the size and laterality of the stones. This success rate was higher than the $88.9 \%$ reported by Al-Hunayan et al. when 27 patients with renal calculi were managed using transperitoneal LPL [10].

In our study, the mean operative time was 100 minutes (Range: 90 - 120 min- 
utes), which was similar to 80 - 150 min mean operative time reported by $\mathrm{Mu}$ jeeburahiman et al. [11]. We experienced a median blood loss of $110 \mathrm{ml}$. This was higher than the $<50 \mathrm{ml}$ experienced by Mujeeburahiman et al. [11]. The mean hospital study in our study was 2.2 days, which was shorter than the 4 days reported by Nouralizadeh et al. who presented the efficacy and possible adverse complications of LPL for the treatment of staghorn stones as an alternative to percutaneous PCNL in a single-surgeon series [12]. This difference could be explained by the relatively low complication rate $(4.84 \%)$ in the current study. Two (3.23\%) patients experienced post-operative colic while 1 (1.61\%) patient developed a fistula as a complication of the intervention. Previous studies have reported complications of laparoscopic pyelolithotomy including prolonged ileus, urinary tract infection, urinary obstruction, urinary leakage, and peritonitis [7] [10] [13]. This study showed that LPL has a high success rate in clearing large $(>20 \mathrm{~mm})$ renal calculi and can be performed with minimal complications.

\section{Conclusion}

Laparoscopic removal of renal and ureteral calculi plays a role in special cases of urolithiasis. Laparoscopic pyelolithotomy provides an innovative and efficient technique for managing calculi of the renal pelvis, especially those with sizes greater than $20 \mathrm{~mm}$. In experienced hands, it can be performed safely; therefore, it could replace open surgery. The benefits of laparoscopic surgery include lower postoperative morbidity, shorter hospitalization, shorter convalescence time, and better cosmetics results.

\section{Acknowledgements}

The authors thank Health Search Association for critically reviewing the manuscript.

\section{Availability of Data and Materials}

The data analyzed in this study are available from the corresponding author upon reasonable request.

\section{Ethics Statement}

Ethical approval was obtained from the institutional review board of the Faculty of Medicine and Pharmaceutical Sciences and the ethics committee of the Centre medico-chirugicaled urologie in Douala, Cameroon. The requirement for informed consent was waived due to the retrospective nature of the study.

\section{Conflict of Interest Statement}

The authors have no conflicting interests to declare.

\section{References}

[1] Assimos, D., Krambeck, A., Miller, N.L., Monga, M., Murad, M.H., Nelson, C.P., et 
al. (2016) Surgical Management of Stones: American Urological Association/ Endourological Society Guideline, PART I. The Journal of Urology, 196, 1153-1160. https://doi.org/10.1016/j.juro.2016.05.090

[2] Bai, Y., Tang, Y., Deng, L., et al. (2017) Management of Large Renal Stones: Laparoscopic Pyelolithotomy versus Percutaneous Nephrolithotomy. BMC Urology, 17, Article No. 75. https://doi.org/10.1186/s12894-017-0266-7

[3] Wang, X., Li, S., Liu, T., et al. (2013) Laparoscopic Pyelolithotomy Compared to Percutaneous Nephrolithotomy as Surgical Management for Large Renal Pelvic calculi: A Meta-Analysis. Journal of Urology, 190, 888-893.

https://doi.org/10.1016/j.juro.2013.02.092

[4] Shah, S.R. (1999) Modified Method for Closure of Transverse Pyelotomy Incision in Gil-Vernet Pyelolithotomy Operation. Techniques in Urology, 5, 106-107.

[5] Salvadó, J.A., Guzmán, S., Trucco, C.A., et al. (2009) Laparoscopic Pyelolithotomy: Optimizing Surgical Technique. Journal of Endourology, 23, 575-578. https://doi.org/10.1089/end.2008.0582

[6] Stein, R.J., Turna, B., Nguyen, M.M., et al. (2008) Laparoscopic Pyeloplasty with Concomitant Pyelolithotomy: Technique and Outcomes. Journal of Endourology, 22, 1251-1256. https://doi.org/10.1089/end.2008.0003

[7] Meria, P., Milcent, S., Desgrandchamps, F., et al. (2005) Management of Pelvic Stones Larger than $20 \mathrm{~mm}$ : Laparoscopic Transperitoneal Pyelolithotomy or Percutaneous Nephrolithotomy? Urologia Internationalis, 75, 322-326.

https://doi.org/10.1159/000089167

[8] Chiva Robles, V., Escalera Almendros, C., Pascual Mateo, C., Rodríguez García, N., García Tello, A. and Berenguer Sánchez, A. (2006) Laparoscopic Pyelolithotomy. Archivos Españoles de Urología, 59, 175-178. https://doi.org/10.4321/S0004-06142006000200008

[9] Wang, J., Yang, Y., Chen, M., et al. (2016) Laparoscopic Pyelolithotomy versus Percutaneous Nephrolithotomy for Treatment of Large Renal Pelvic Calculi (Diameter > 2 cm): A Meta-Analysis. Acta Chirurgica Belgica, 116, 346-356. https://doi.org/10.1080/00015458.2016.1181312

[10] Al-Hunayan, A., Abdulhalim, H., El-Bakry, E., et al. (2009) Laparoscopic Pyelolithotomy: Is the Retroperitoneal Route a Better Approach? International Journal of Urology, 16, 181-186. https://doi.org/10.1111/j.1442-2042.2008.02210.x

[11] Mujeeburahiman, M. and Vipin, C. (2018) Laparoscopic Pyelolithotomy as a Monotherapy for the Management of Intermediate-Sized Renal Pelvic Stones. Urology Annals, 10, 254-257. https://doi.org/10.4103/UA.UA_80_17

[12] Nouralizadeh, A., Simforoosh, N., Soltani, M.H., et al. (2012) Laparoscopic Transperitoneal Pyelolithotomy for Management of Staghorn Renal Calculi. Journal of Laparoendoscopic \& Advanced Surgical Techniques, 22, 61-65. https://doi.org/10.1089/lap.2011.0302

[13] Nambirajan, T., Jeschke, S., Albqami, N., et al. (2005) Role of Laparoscopy in Management of Renal Stones: Single-Center Experience and Review of Literature. Journal of Endourology, 19, 353-359. https://doi.org/10.1089/end.2005.19.353 